\title{
Polarization phenomena in coherent excitation of atomic systems: an overview of results
}

\author{
N. V. Sytenko \\ Mozhaisky Military Space Academy 13, Zhdanovskaya, St. Petersburg, 197198, Russia \\ vka@mil.ru
}

DOI 10.17586/2220-8054-2021-12-6-690-696

\begin{abstract}
The article gives an overview of the main theoretical and experimental studies of the phenomena caused by the formation of coherent states coherent population trapping, electromagnetically induced transparency, and laser generation without inversion.
\end{abstract}

Keywords: $\Lambda$-scheme, Liouville von Neumann equation, dark state, Hanle effect, Rabi frequency.

Received: 10 October 2021

Revised: 27 November 2021

\section{Introduction}

Many phenomena are known to be conditioned by the formation of coherent states. They include laser generation itself and the phenomena resulting from laser generation - coherent population trapping and electromagnetically induced transparency.

The phenomenon of coherent population trapping (CPT) was discovered in the 1980s and, therefore, it has been studied comprehensively. However, the studies on CPT gave impetus for research into the phenomena associated with coherent trapping, which led to the discovery of new phenomena as well as to the search for practical applications of CPT, such as frequency stabilization and ultrahigh resolution spectroscopy, lasers without inversion, obtaining "slow" light, and ultra-deep cooling of atoms, the phenomenon that may lead to the creation of electronic memory. In addition, it was proposed to use CPT as a basis for spatial superlocalization of atoms for dimensions that are much smaller than the light wavelength in caustics of inhomogeneously polarized wave fronts; also, a method for diagnosing the magnetic field in plasma was developed, etc. Since the research began, coherent population trapping has been showing itself in an increasing number of experimental situations. The "side effects" of this phenomenon lead to unexpected and surprising consequences, some of which are not fully understood.

\section{Coherent population trapping}

In the late 1990s, interest in the behavior of three-level systems in the fields of two electromagnetic waves increased. The research showed that three-level systems (in the general case, multilevel systems) cannot always be excited to the upper state. This phenomenon was first described in [1], [2]; the authors experimentally detected the disappearance of absorption in an ensemble of sodium atoms was when it was irradiated by a laser field. Subsequently, this phenomenon was called "coherent population trapping". Further studies, the results of which are partially given in [3], showed that during excitation according to the $\Lambda$-scheme (see Fig. 1), special states are generated in the system that do not interact with the external electromagnetic field; these states arise at certain ratios between frequency detuning in each wave used for excitation.

In [4], a three-level system comprising two lower levels of the superfine structure of the ${ }^{87} \mathrm{Rb}$ atom ground state $5^{2} S_{1 / 2}$ and a lower level of the superfine structure of $5^{2} P_{1 / 2}$ state of that atom is considered. The authors compare the theoretical and experimental time dependences of absorption arising from a sudden change in the sign of the frequency detuning. The theoretically obtained dependence is accompanied by oscillations whose frequency increases with time, which agrees with the experimental results.

Consideration of the CPT phenomenon, not related to the solution of systems of differential equations, but based only on the symmetry properties, is presented in [5], where a system of three levels $a, b$ and $c$, and level $b$ is populated from levels $a$ and $c$ by the $\Lambda$-scheme. This paper shows that if the wave function of a system is expanded by eigenfunctions of states $|a\rangle,|b\rangle$ and $|c\rangle$, then, based on the symmetry properties of the differential equations for products of the form $c_{i} \frac{d}{d t} c_{i}, i=a, b, c$, where $c_{a}, c_{b}$ and $c_{c}$ are expansion coefficients, we can obtain a relation resembling the hyperboloid equation with complex coefficients determined by dipole moments. Further, using normalization of expansion coefficients, the relations linking the squares of the population modulus of states $|a\rangle$ and $|c\rangle$ through the functions describing the fields in the shoulders of the $\Lambda$-scheme and the population of state $|b\rangle$ are obtained in the paper. Finally, 


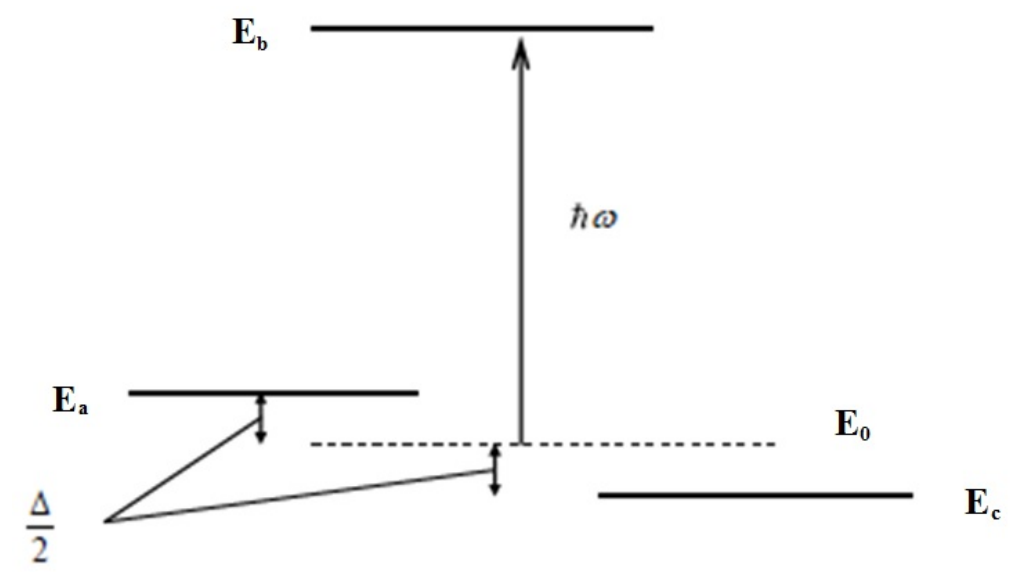

FIG. 1. $\Lambda$-scheme

assuming that the envelopes of these fields are described by Gaussian packets, the geometrical interpretation of the experimental results is performed.

It is essential that in experiments, the CPT phenomenon is often recorded in the form of the Hanle effect, which manifests itself in a sharp decrease in absorption depending on the magnitude of the magnetic field strength in the vicinity of the zero value of this field. Such a dip is often called "black resonance". The black resonance curves were observed in [6], [7], where transitions between the superfine levels of the sodium atom in a magnetic field were studied. In [7], using numerical integration of the Liouville von Neumann equation for closed and open systems, it was shown that in the general case, the width of the black resonance depends on the magnetic field $H$ and the integration time (in the authors' terminology, "interaction time") $t_{\text {fin }}$ or, in other words, $I=I\left(H, t_{f i n}\right)$. Note that the width of resonance decreases with an increase in interaction time. The authors explain it by the population loss of the open system in the process of evolution. Thus, it follows from this work that an increase in the interaction time leads to dark resonance narrowing, and the main contribution to the intensity is made by the neighborhood of point $H=0$, where the absence of absorption leads to the absence of the population loss. In a closed system, where the population remains, $I\left(H, t_{f i n}\right)$ does not depend on time.

In [8], a method for diagnosing the magnetic field in plasma is proposed. It is based on the following idea: if the direction of the magnetic field coincides with the direction of the propagating electromagnetic wave $(\vec{H} \| \vec{k})$, the electric field will induce dipole moments in the direction perpendicular to the field, leading to transitions between magnetic sublevels with changes in the magnetic quantum number $\Delta m= \pm 1$ and transitions with frequency $2 \omega_{L}$. If $\vec{H}$ and $\vec{k}$ directions do not coincide, then the system will have transitions with frequencies $\omega_{L}$ and $2 \omega_{L}$. This circumstance allows us to judge about the magnetic field direction by the with respect to the radiation intensities at the frequencies $\omega_{L}$ and $2 \omega_{L}$.

In [9], where, using the ${ }^{87} \mathrm{Rb}$ as an example, the influence of system parameters on the character of the three-level system transition to the CPT state is considered. It is shown that at high Rabi frequencies, the system's transition to the CPT state is accompanied by oscillations. In the opposite case of small Rabi frequencies, the system reaches this state smoothly, after a short transient process.

Because of superfine splitting, each of the two lower levels of the ${ }^{87} \mathrm{Rb}(I=3 / 2)-{ }^{2} \mathrm{~S}_{1 / 2}$ and ${ }^{2} P_{1 / 2}$ 一, splits into two levels characterized by the total moments $F_{1}=1$ and $F_{2}=2$. If we now place such a system in a magnetic field and excite it with light polarized along the left-hand circle of the upper level $F_{1 e}=2$ from the two lower levels $F_{2 g}=2$ and $F_{1 g}=1$, then a transition $M_{F_{1 g}}=-2 \rightarrow M_{F_{2 e}}=-1$ is formed, as well as a chain of three $\Lambda$-schemes:

$$
\begin{gathered}
\Lambda_{1}: M_{F_{1 g}}=0 \rightarrow M_{F_{2 e}}=-1 ; M_{F_{2 g}}=0 \rightarrow M_{F_{2 e}}=1 ; \\
\Lambda_{2}: M_{F_{1 g}}=0 \rightarrow M_{F_{2 e}}=1 ; M_{F_{2 g}}=0 \rightarrow M_{F_{2 e}}=1 ; \\
\Lambda_{3}: M_{F_{1 g}}=1 \rightarrow M_{F_{2 e}}=2 ; M_{F_{2 g}}=1 \rightarrow M_{F_{2 e}}=2 .
\end{gathered}
$$

If we now modulate the applied magnetic field according to the harmonic law $\cos \left(\frac{4 \pi}{T} t+\phi\right)$, we will find $\Lambda_{2}$ system in the CPT state at point $t=\frac{T}{2}$. Due to the shift of magnetic sublevels in the magnetic field, $\Lambda_{1}$ and $\Lambda_{3}$ systems will reach the CPT state at a time somewhat later than $\Lambda_{2}$. The transition of $\Lambda_{2}$ to this state will be accompanied by oscillations, which, according to [9], are caused by a redistribution of the population in $\Lambda_{2}$ system. In the case 
of an increase in the detuning, systems $\Lambda_{1}$ and $\Lambda_{3}$ first go to the CPT state at certain but different times, and at very significant detuning, these two systems can be considered as one that goes to the CPT state near the point where the amplitude of the magnetic field modulation becomes zero.

Papers [10], [11], and [12] consider the effect of the field phases involved in the excitation process of multilevel systems closed in the excitation process, i.e., when the excitation process depends on the characteristics of only a pair of neighboring levels $\vec{E}=\sum_{i} \vec{e}_{i} \varepsilon_{i} \cos \left(\omega_{i} t+\phi_{i}\right)$, where $\vec{e}_{i}$ is the polarization field vector with amplitude $\varepsilon_{i}$, frequency $\omega_{i}$, and phase $\phi_{i}$, which has the sense of the excitation frequency tuning. It is shown that if the condition $\omega_{1 n}=\sum_{i=1}^{n-1} \omega_{i, i+1}$ of system closure in the excitation process is fulfilled, the solution of the system of density matrix equations depends on some total phase $\Phi=\phi_{n, 1}-\sum_{i=1}^{n-1} \phi_{i+1, i}$. The results of theoretical studies are compared with those of the experiment in [4], where the absorption oscillations were observed with a sharp change in the detuning in the radio-frequency field arm. The experiment showed that the absorption signal oscillation frequency increased with an increase in the tuning, which agrees with the theoretical prediction.

In all of the above-mentioned publications, the width of the signal used for excitation was assumed to be much narrower than the width of the levels included in the excited system. In the opposite case, when considering the CPT problem, it should be assumed that the system in question interacts with a field the phases of which are random quantities. The presence of random phases leads to the fact that the system of equations for the density matrix contains random quantities and, therefore, is stochastic. Consequently, its solution does not give the quantities themselves, but only their values averaged over the phase distribution [13].

A theoretical study on the emission of $6 s 2^{1} S_{0} \rightarrow 6 s 6 p^{1} P_{1}$ transition in an ensemble of ${ }^{137} \mathrm{Ba}$ and ${ }^{138} \mathrm{Ba}$ atoms in the presence of a magnetic field used to separate the Zeeman sublevels is carried out in [14]. In this case, the system of ${ }^{1} P_{1}$ state sublevels forms the upper state, while a similar set of ${ }^{1} S_{0}$ state sublevels forms the lower state. All levels between the upper and lower states form a reservoir where the upper state decays radiatively. Both $\sigma$ and $\pi$ radiations were used for excitation. This paper also considers the shapes of the resonances resulting from the transitions from various Zeeman sublevels of the excited state to similar sublevels of the ground state.

Reference [15] considers the effect of a strong magnetic field on the polarization characteristics of the radiation of a multilevel system whose set of lower levels is populated from the ground state by axially symmetric excitation, while the upper levels are populated from the lower levels in a coherent manner. Specific calculations were made for $2 p^{3} P_{j}-3 s^{3} S_{1}$ transition of the helium atom. Using the numerical solution of the Liouville von Neumann equation, it is shown that the system reaches the CPT state when the laser frequency becomes quite significant. At smaller values of detunings, a population-type ordering of angular momentums is induced on the excited state of the system, whereas an alignment-type ordering is absent. When the direction of excitation anisotropy makes a right angle with the magnetic field's direction, the system reaches the CPT state, i.e., the population and, consequently, the alignment of the system are zeroed out.

The description of the CPT phenomenon in complex polyatomic systems is, at least from the computational point of view, a rather complicated problem. When considering such problems, one has to resort to various approximations. For example, in [16], when considering a three-level system consisting of $6^{2} P_{1 / 2}, 6^{2} P_{3 / 2}$ and $6^{2} S_{1 / 2}$ levels of $T l$ atom in the presence of a magnetic field, the wave function of the system was defined as an expansion in the eigenfunctions of these states. The resulting system of differential equations is solved in the resonance approximation, which reduces the problem to the solution of a system of differential equations with constant coefficients. As a result, the time dependence of the squares of the modulus of the expansion coefficients, the populations of the states in question, was obtained.

To conclude the discussion of the CPT phenomenon, it should be noted that in [17], where the problem of observing the CPT phenomenon in semiconductors was discussed theoretically using numerical integration of the Bloch equations, it was shown that despite the difficulties associated with the band structure and the strong multiparticle effects associated with excitons, signs of dark states can also be observed in these media.

\section{Electromagnetically induced transparency}

A phenomenon related to CPT is electromagnetically induced transparency (EIT), which was discovered in 1991 [18]. It consists in disappearance of absorption of a weak test field in one arm of the $\Lambda$-scheme, while a strong binding field acts in the other arm. Under the action of these two fields, the system becomes practically transparent with respect to the test field if the frequency difference of the fields acting in both shoulders coincides with the frequency difference of the transitions. From a computational point of view, the EIT problem is easier than the similar CPT problem, since in the latter case, the system's evolution can be found by numerical solution of the system of differential equations for 
the density matrix. In the EIT problem it is initially assumed that the binding field is much stronger than the test field, for which the Liouville von Neumann system of equations is to be solved based on perturbation theory.

A considerable number of works are devoted to the EIT phenomenon (see review [19]). In addition, Reference [20] experimentally and theoretically illustrates the disappearance of absorption in $5^{2} S_{1 / 2}(F=1) \leftrightarrow 5^{2} P_{1 / 2}(F=2)$ transition in an ensemble of ${ }^{87} \mathrm{Rb}$ atoms, while a strong binding field acts in $5^{2} S_{1 / 2}(F=2) \leftrightarrow 5^{2} P_{1 / 2}(F=1)$ shoulder. Reference [21] considers the EIT phenomenon in an optically dense medium, which is particularly interesting because both the absorption and dispersion properties of the medium can be controlled by the phase of the laser beam, which makes it possible to control the properties of the propagating radiation more efficiently than in a conventional experiment on EIT.

The system of three superfine levels of ${ }^{87} \mathrm{Rb}$ atom is considered in [22]. The test beam corresponded to the $5^{2} S_{1 / 2}(F=1) \leftrightarrow 5^{2} P_{1 / 2}(F=1)$ transition, and the binding one, to the $5^{2} S_{1 / 2}(F=2) \leftrightarrow 5^{2} P_{1 / 2}(F=1)$ transition. If we do not consider the influence of magnetic sublevels, the system under consideration is a classical $\Lambda$ scheme. In the case when this system is in the CPT state, it can be argued that, eventually, only $5^{2} S_{1 / 2}(F=1)$ level will be populated, since the Rabi frequency in the arm containing the binding beam is larger than the one in the arm containing the trial beam. The paper shows that the consideration of Zeeman sublevels has a strong influence on the behavior of the system, which is manifested by the fact that the set of Zeeman sublevels $5^{2} P_{1 / 2}(F=1)$ and $5^{2} S_{1 / 2}(F=2)$ forms a system in the CPT state, and all the population accumulates mainly at the $5^{2} S_{1 / 2}(F=2)$ level. Moreover, the CPT state is achieved at relatively weak binding fields and it weakly depends on detuning.

Along with the EIT phenomenon, which consists in enlightening the medium due to coherence, there is an opposite, in some sense, phenomenon - electromagnetically induced absorption (EIA) [23], [24], [25]. A prerequisite condition for the EIA is a strong difference in the excitation intensities of the test and binding beams. In [25] it is shown that the necessary conditions under which the EIA emerges are the following:

1. the quantum numbers of the upper and lower states $e$ and $g$-respectively $F_{e}$ and $F_{g}$-differ by one;

2. $g \rightarrow e$ transition is closed;

3. the ground state is degenerate, i.e., $F_{g}>0$.

In [24] and [25], the EIA phenomenon is described in detail. In [24], the $5^{2} S_{1 / 2}(F=3) \rightarrow 5^{2} P_{1 / 2}(F=4)$ transition of ${ }^{87} \mathrm{Rb}$ atom is studied both experimentally and theoretically. In [25], the results of the experiment on the observation of resonance from the $5^{2} S_{1 / 2}(F=3) \rightarrow 5^{2} P_{3 / 2}$ transition are analyzed.

In [26], for a mixture of ${ }^{87} \mathrm{Rb}$ atoms and a buffer gas, neon, in particular, the effect of a test beam, polarized linearly and corresponding to the $F_{0}=2 \rightarrow F=1$ transition, on the EIT signal was studied. The Hanle effect, which implies the presence of a sharp maximum in the bandwidth at zero magnetic field, was used to record the system's enlightening by the test beam. The observations showed that the EIT signal has the form of a Lorentz curve if the laser beam has the form of a broad Gaussian curve or has a low intensity. A similar situation is observed if the laser beam is U-shaped with the same width as the aforementioned Gaussian curve. For a narrow Gaussian beam or a beam of high intensity, the EIT signal has a non-Lorentzian shape.

In [27], the EIP phenomenon was considered experimentally and theoretically on a mixture of ${ }^{87} \mathrm{Rb}$ and ${ }^{85} \mathrm{Rb}$ isotopes. The theoretical consideration was based on a system of six superfine levels: two superfine structure levels of the ground ${ }^{2} S_{1 / 2}$ state and four superfine structure levels of the first excited state ${ }^{2} P_{3 / 2}$. The test and binding fields in this work are directed toward each other and have opposite linear polarizations. As a result of the experiment, the absorption dependences of the test field on the binding field detuning were obtained. The experimental results are compared with the data of numerical calculations made in the framework of the Liouville von Neumann solution. In so doing, nonuniformity of the populating levels of the superfine structure caused by the Doppler effect is taken into account. The paper also considers the possibility of describing the system within the framework of the traditional three-level excited by the $\Lambda$-scheme. It is shown that the resulting half-width of the EIT resonance strongly depends on the velocities and, after averaging over the velocities, can be used for the qualitative characteristics of the system under consideration.

An interesting way of creating coherent states is described in [28], which considers a system formed by three levels of the $P b$ atom: $6 p 7 p[1 / 2,3 / 2]_{1}$ (level $\left.c\right), 6 s 7 s[1 / 2,1 / 2]_{1}$ (level $\left.b\right), 6 p^{2}[1 / 2,1 / 2]_{0}($ level $a$ ). A strong field binds $b$ and $c$ levels, whereas the frequency of the test field is somewhat less than the transition frequency between $b$ and $a$ levels. The binding field forms a coherent state from $|b\rangle$ and $|c\rangle$ states. The experimental conditions are such that the considered levels are collisionally broadened, and the collisions broaden $b$ and $a$ levels to a greater extent. The interaction energy of atoms of the same element in the same states (resonance collisions) is inversely proportional to the third degree of inter-nuclear distance, whereas the similar value for collisions of the same atoms in different states is inversely proportional to the sixth degree of internuclear distance. Therefore, the Lorentzian lineshape corresponding to the $a \leftrightarrow b$ transition will widen and overlap with a similar lineshape corresponding to the $c \leftrightarrow b$ transition . 
To conclude the overview of the works devoted to the EIT phenomenon, it is worth noting that in solids, where states are strongly broadened, the creation of coherent states may involve serious difficulties. These difficulties were partially overcome in [29], where absorption disappeared in a ruby crystal under the action of an external field.

\section{Laser generation without inversion}

Another phenomenon associated with induced coherence is laser generation without inversion. The assumptions about the feasibility of obtaining this phenomenon were first made in [30] and [31]. In [30], the authors point to a possibility of amplifying an ultrashort pulse in the $\Lambda$-scheme due to low-frequency coherence. In [31], the problem of the evolution of the system in question was solved in the framework of the Schrödinger equation, i.e., the wave function of the system was expanded by the system of wave functions of the states under consideration with coefficients $a_{1}, a_{2}$, $a_{3}$. The absorption coefficient $W_{a b} \sim-\frac{d}{d t}\left|a_{1}\right|^{2}$ was calculated under the initial condition of $a_{1}=1$. Similarly, the transmission coefficient was calculated under the initial condition of $a_{2}=1$.

The possibility of inversionless generation is considered in monograph [32]. Here, at arbitrary population of the levels of a three-level system at the initial moment of time, the Liouville von Neumann equation for the density matrix is solved in approximation linear on Rabi frequencies. It follows that one can choose the parameters of the problem so that the absorption will become zero.

The fundamental role of low-frequency coherence in laser generation without inversion was demonstrated in [33], where an ensemble of Sm atoms is considered. Its lower state has a total momentum $j=1$, and the upper state has a total momentum $j=0$. Due to Zeeman splitting, the superimposed magnetic field forms a four-level system. A sequence of picosecond pulses polarized in the right and left circles was used to induce low-frequency coherence at the Zeeman sublevels of the lower state according to the scheme $j=1 ; m=-1 \leftrightarrow j=0 ; m=0 \leftrightarrow j=1$; $m=1$. After passing through the sequence of pulses, at a moment in time consistent with the sign and magnitude of the induced low-frequency coherence, a linearly polarized short pulse ( $\pi$-pulse) is fed to provide the population of the upper state according to the scheme $j=1 ; m=0 \leftrightarrow j=0 ; m=0$. However, the population of the upper state was always less than the population of the lower state. The authors observed an abrupt increase in the signal if the $\pi$-pulse was formed at a time when the low-frequency coherence was maximal (the latter oscillated with the doubled Larmor frequency), and no signal enhancement was observed if the $\pi$-pulse was formed when this coherence was absent.

The feasibility of laser generation without inversion is discussed in many works (for example, [34], [35], [36]). However, Zibrov and others were the first to observe this phenomenon experimentally. In their work [37], the authors consider set of four levels of the ${ }^{87} \mathrm{Rb}$ atom superfine structure: two levels of the superfine structure of the ground state ${ }^{2} S_{1 / 2}$ with $F=1$ (level $b$ ) and $F=1$ (level $b^{\prime}$ ), the first excited state ${ }^{2} P_{1 / 2}$ with $F=2($ level $a$ ), and one of the levels of the superfine structure of ${ }^{2} P_{3 / 2}$ state with $F=2$ (level $c$ ). Levels $c$ and $b$ are bound by a strong binding field with the Rabi frequency $\Omega_{c}$, whereas levels $a$ and $b$ are bound by a weak test field. Since the set of $a, b, c$ levels form a $V$-scheme, the joint action of the test and binding fields results in the highest population at $b^{\prime}$ level. If we introduce another field (pumping field) linking the levels $b^{\prime}$ and $a$, then the population balance will change due to the radiative decay of $a$ level into $b$ and $b^{\prime}$ levels.

When solving the system of equations for the density matrix in accordance with perturbation theory, being limited to the zero order by the test field, the relation for the absorption coefficient in $a-b$ shoulder is obtained:

$$
\chi \sim-\frac{\left(\rho_{a a}^{(0)}-\rho_{b b}^{(0)}\right)+\frac{\left|\Omega_{c}\right|^{2}}{\gamma_{a c} \gamma_{b c}}\left(\rho_{b b}^{(0)}-\rho_{c c}^{(0)}\right)}{\gamma_{a c}+\frac{\left|\Omega_{c}\right|^{2}}{\gamma_{a c}}} .
$$

The formula shows that negative absorption requires either a population inversion- $\rho_{a a}^{(0)}>\rho_{b b}^{(0)}-$ or, in the case of large Rabi frequencies, $\rho_{b b}^{(0)}>\rho_{c c}^{(0)}$, which can be achieved for good ratios between the radiative decay efficiencies.

In that study, the authors confirmed both theoretically, using the numerical solution of the system of the Liouville von Neumann equations for the density matrix, and experimentally the presence of the test field frequency interval, at which the absorption coefficient becomes negative, that is, there is a generation.

Work [38] is similar to [37] with the difference that in the first one, the authors use the $\Lambda$ - scheme formed by two levels of the superfine structure of the sodium atom in ${ }^{2} S_{1 / 2}$ state with $F=1$ and $F=2$, as well as ${ }^{2} P_{1 / 2}$ level of the first excited state of this atom to experimentally confirm generation without inversion. In this work, a strong binding field acts between $F=2$ levels of ${ }^{2} S_{1 / 2}$ state and $F=1$ level of ${ }^{2} P_{1 / 2}$ state. This field, together with a very weak test field binding the superfine structure levels of ${ }^{2} S_{1 / 2}$ and ${ }^{2} P_{1 / 2}$ states with $F=1$, leads to the emergence of EIT resonance. The authors experimentally show that at a frequency close to the EIT frequency, the test signal in the 
system is amplified, which holds for a very short period of time at the same frequency as the test field. The system was placed in a ring resonator, wherein laser generation caused by vacuum fluctuations was observed.

In [39], the feasibility of laser generation without inversion at fine levels of the helium atom is considered. The approach to the generation problem in this work is fundamentally different from that proposed in [37], [38]. Within the framework of the built model, the possibility of generation is shown by reducing the broadening of the lower levels of the $\Lambda$-scheme. According to the $\Lambda$-scheme, excitation was carried out from the excited $2^{3} P_{1}$ and $2^{3} P_{2}$ helium atom states, which made it possible to avoid a significant broadening of the lower levels due to their interaction with the buffer gas. It is shown that although the frequency in one arm of the $\Lambda$-scheme is a hundred times greater than in the other, no less strong polarization field effect is obtained even at equal Rabi frequencies. In the paper it is noted that creation and maintenance of population at levels $2^{3} P_{1}$ and $2^{3} P_{2}$ in practice is most likely to involve certain difficulties.

In [40], an approach proposed to obtain laser generation without inversion does not require a quantum superposition of states in the active medium. The authors show that with periodically varying pumping, the generation is achievable even when the population inversion of the active medium is negative. This is possible due to the fact that the laser in the strong coupling mode is a nonhermetic system with an exceptional point existing at the negative value of the population inversion. It is shown that because of the nonorthogonality of the eigenstates near the exceptional point, a change in the pumping power over time can lead to laser parametric instability and excite autoscillations of the electromagnetic field. These oscillations take place even when the population inversion of the active medium is negative.

In standard lasers, light amplification requires a population inversion between the upper and lower states in order to break the reciprocity between absorption and stimulated emission. However, in a medium in a certain superposition state, quantum interference can completely suppress absorption, leaving the stimulated emission intact, which opens the possibility of radiation without inversion. Such a situation is considered in [41]. The authors show that radiation without inversion occurs naturally during the propagation of intense femtosecond laser pulses in air. It is caused by a combination of molecular ionization and molecular alignment, which are inevitable in intense light fields. This effect may allow for broadband radiation in many molecular gases without inversion, opening up unusual possibilities for remote sensing.

\section{References}

[1] Alzetta G., Moi L., Orriols G. Nonabsorbtion hyperfine resonance in sodium vapor irradiated by a Multimode dye-laser. Novo. Chimento, 1979, 52(2), P. 209-217.

[2] Alzetta G., Gozzini A., Moi L., Orriols G. An experimental-method for the observation of Rf transitions and laser beat resonances in oriented Na vapor. Novo. Chimento, 1976, 36, P. 5.

[3] Agapiev B.D., Gorny I.B., Matisov B.G., Rozhdestvensky Yu.V. Coherent population trapping in quantum systems. UFN, 1993, 183(9), P. $1-36$.

[4] Park Song Jong, Cho Hyuck, Kwon Taeg Yong, Lee Ho Seong. Transient coherence oscillation induced by a deturned Raman field in a rubidium $\Lambda$-system. Phys. Rev. A, 2004, 69, P. 023806.

[5] Zhang G.P. Hidden identity in a generic $\Lambda$-system application to coherent population trapping. J. Phys. B, 2013,46 , P. 035504.

[6] Renzoni F., Maichen W., Windholz L., Arimondo E. Coherent population trapping with losses observed on the Hanle effect of the $D_{1}$ sodium line. Phys. Rev. A, 1997, 55, P. 3710-3718.

[7] Renzoni F., Arimondo E. Population-loss-ed narrowing of the dark resonance. Phys. Rev. A, 1998, 58(6), P. 4717-4722.

[8] Akhmedzhanov R., SnplaceZlensky SnI., Kuznetsova E. Magnetic field diagnostics of plasma based on coherent population trapping: theory and experiment. Phys. Rev. E, 2004, 69, P. 036409.

[9] Margalit L., Rosenbluh M., Wilson-Condon A.D. Coherence-population-trapping transients indused by the magnetic field. Phys. Rev. A, 2012, 52, P. 063809.

[10] Kosachev D., Matisov B.G., Rozhdestvensky Yu.V. The coherent phenomena in multiplet systems with closed interaction contour. J. Phys. B, 1992, 25, P. 2473-2488.

[11] Kosachev D., Matisov B.G., Rozhdestvensky Yu.V. Coherent population trepping: sensitivity of an atomic system to the relative phase of exciting fields. Opt. Commun, 1991, 85, P. 209-212.

[12] Maichen W., Renzoni F., SnplaceMazets SnI., Korsunsky E., Windholz L. Transient coherent population trepping in a closed loop unteraction scheme. Phys. Rev. A, 1996, 53(5), P. 3444-3453.

[13] Stenholm S. Fundamentals of laser spectroscopy. Nauka, CityplaceMoscow, 1986, 280 p.

[14] Kai-Shue-Lam. Coherent laser excitation of ${ }^{137}$ Ba and ${ }^{138}$ Ba. Phys. Rev. A, 1992, 45(5), P. 3084-3092.

[15] Petrashen A.G., Sytenko N.V. Polarization phenomena during coherent excitation in the presence of a strong magnetic field. Nanosystems: physics, chemistry, mathematics, 2012, 3(5), P. 62-69.

[16] Karagodova T.Ya. The influence of the orientation and strength of a magnetic field on the coherent population trapping in the $\Lambda$-system. Optics and Spectroscopy, 2002, 93(2), P. 194-197.

[17] Lindberg M., Binder R. Dark states in coherent semiconductor spectroscopy. Phys. Rev. Lett. A 45, 1995, 75(7), P. 1403-1406.

[18] Boller K-J., Imamoglu A., Harris S.E. Observation of electromagnetically induced transparency. Phys. Rev. Lett., 1991, 66(20), P. 2593-2596.

[19] Fleischhauer M. Electromagnetically induced transparency: Optics in coherent media. Rev. Mod. Phys., 2005, 77, P. 633-673.

[20] Yong-qing Li, Xiao Min Electromagnetically induced transparency in a three-level $\Lambda$-type system in rubidium atoms. Phys. Rev. A, 1995, 53(4), P. 2703-2706. 
[21] Korsunsky E.A., Leinfellner N., Hass A., Baluschev S., Windholz L. Phase-dependent electromagnetically induced transparency. Phys. Rev. A, 1999, 59(3), P. 2303-2305.

[22] Hong Yuan Ling, Yong Oing Li, Min Xiao Coherent population trapping and electromagnetically induced transparency in multi-zeemansublevel atoms. Phys. Rev. A, 1996, 53(2), P. 1014-1026.

[23] Valente D., Failache H., Lezmana A. Conparative study of the transient evolution of Hanle electromagnetically induced transparency and absorption resonance. Phys. Rev. A, 2002, 53(2), P. 023814.

[24] Akulshin A.M., CityplaceBarreiro S., Lezama A. Electromagnetically induced absorption and transparency due to the resonant excitation of quasidegenerate levels of Rbvapor. Phys. Rev. A, 1998, 57(4), P. 2996-3002.

[25] Lezama A., CityplaceBarreiro S., Akulshin A.M. Electromagnetically induced absorption. Phys. Rev. A, 1999, 59(6), P. 4732-4735.

[26] Nicolic S.N., Radonjic M., Krmpot A.J., CityplaceLucic StateN.M., Zlatkovik B.V. Effects of laser beam profile on Zeeman electromagnetically induced transparency in the Rb buffer gas cell. J. Phys. B: At. Mol. Opt. Phys., 2013, 46, P. 075501.

[27] Bhattacharyya D., Ghosh A., Bandyopadhyay A., Saha S., De S. Observation of electromagnetically induced transparency in six-level Rb atoms and theoretical stimulation of the observed spectra. J. Phys. B, 2015, 48, P. 175503.

[28] Field J.E., Hahn K.H., Harris S.E. Observation of electromagnetically induced transparency in collisionally broadened lead vapor. Phys. Rev. Lett., 1991, 67(22), P. 3062-3065.

[29] Yang Zhao, Cunckal Wu, Byoung-Seung Ham, Kim M.K., Microwave induced transparency in Ruby. Phys. Rev. Lett., 1997, 79(4), P. 641-644.

[30] Kocharovskaya O.A., Khanin Y.I. Coherent amplification of an ultrashort pulse in a three-level system without population inversion. Letters to ZhETF, 1988, 48, P. 581-583.

[31] Harris S.E. Laser without inversion: interference of lifetime broaded resonances. Phys. Rev. Lett., 1989, 62(9), P. 1033-1036.

[32] Scully M.O., Zubairi M.S. Quantum optics. Fizmatlit, placeCityMoscow, 2003, 510 p.

[33] Nottelman A., Peters S., Lange W. Inversionless amplification of picosecond pulses due to Zeeman coherence. Phys. Rev. Lett., 1993, 70(12), P. $1783-1786$.

[34] Imamoglu A., Harris S. Lasers without inversion: interference of dressed life-time broadened state. Opt. Lett., 1989, 14(24), P. 1033-1036.

[35] Agarwal G.S. Inhibition of spontaneous emission noise in lasers without inversion. Phys. Rev. Lett., 1991, 67(8), P. 980982.

[36] Gheri K.M., Walls D.F. Sub-Shot-Noise laser without inversion. Phys. Rev. Lett., 1992, 68(23), P. 3428-3431.

[37] Zibrov A.S., Lukin M.D., Niconov D.E., Holiberg L.,Scully M.O., Velichansky V.L., Robinson H.G. Experimental demonstration of laser oscillation without population inversion via quantum interference in Rb. Phys. Rev. Lett., 1995, 75(8), P. 1499-1501.

[38] Pandanabandu G.G., Welch G.R., Shubin I.N., Fry E.S., Nikonov D.E., Lukin M.D., Scully M.O. Laser oscillation without population inversion in sodium atomic beam. Phys. Rev. Lett., 1996, 76(12), P. 2053-2056.

[39] Petrashen A.G., Sytenko N.V. Laser generation without inversion on the fine levels of the helium atom. Nanosystems: physics, chemistry, mathematics, 2016, 7(2), P. 384-393.

[40] Doronin I.V, Zyablovsky A.A., Andrianov E.S., Pukhov A.A., Vinogradov A.P. Lasing without inversion due to parametric instability of the laser near the exceptional point. Phys. Rev. A, 2019, 100, P. 021801.

[41] Lytova M., Morales F., Haessler S., Smirnova O., Spanner M., Ivanov M. Rotational quantum beat lasing without inversion. Optica, Optical Society of placecountry-regionAmerica - OSA Publishing, 2020, 7(6), P. 586-592. 